\title{
Morphological study of rugae palatinae in Sudanese Nubians
}

\author{
A.A. Ahmed 1,2 , A. Hamid ${ }^{3}$ \\ ${ }^{1}$ Department of Basic Medical Sciences, College of Medicine, King Saud bin Abdulaziz University for Health Sciences, \\ Riyadh, Kingdom of Saudi Arabia \\ ${ }^{2}$ Anatomy Department, Faculty of Medicine, University of Khartoum, Khartoum, Sudan \\ ${ }^{3}$ Department of Anatomy, Faculty of Dentistry, University of Medical Sciences and Technology, Khartoum, Sudan
}

[Received 23 October 2014; Accepted 17 December 2014]

Background: The aim of this study was to identify and compare the various morphological characteristics of rugae palatinae in males and females of adult Sudanese Nubians and to assess the existence of side asymmetry. Furthermore, the possibility of assigning sex utilising dimensions, shapes, and orientations was explored using logistic regression.

Materials and methods: In all, 100 maxillary casts were equally distributed between the sexes. All subjects belonged to a similar age group, ranging from 18 to 23 years of age, and were examined for rugae palatinae dimensions, shapes, and orientations for the purpose of assessing sexual dimorphism and side symmetry. Results: The most dominant were the primary rugae, and the most prevalent shapes were the wavy, curved, and straight in both sexes. The dominant direction of rugae was the forward-directed. Furthermore, while there was no sexual dimorphism in rugae orientation, primary rugae and converging unified rugae exhibited significant differences between the sexes. Significant asymmetry was observed in females' primary and wavy shaped rugae, a diverging unified shaped was found in males, and forward-and backward-directed rugae were found in both sexes.

Conclusions: This study provided reference data regarding the rugae palatinae morphology among Sudanese population. A predictive value of $60 \%$ was obtained when assigning sex using dimensions and orientations, and a predictive value of $65 \%$ was determined when using only shapes. Accordingly, using the rugae palatinae to accurately estimate sex among Sudanese Nubians is not recommended unless it is the only means available. (Folia Morphol 2015; 74, 3: 303-310)

Key words: palatal rugae, palate, casts, identification, regression, morphology

\section{INTRODUCTION}

Plicae palatinae transversae or rugae palatinae among humans are asymmetrical, irregular mucosal ridges arranged in a transverse direction on either side of the median raphe palati in the anterior part of the palatum behind the papilla incisiva $[14,27]$. Their evolutionary development and applications have been a focus of interest in anatomy, anthropology, genetics, forensic odontology, prosthodontics, and orthodontics. The rugae were described in anatomy texts for the first time by J.B. Winslow in

Address for correspondence: Dr A.A. Ahmed, Department of Basic Medical Sciences, College of Medicine, King Saud bin Abdulaziz University for Health Sciences, Mail Code: 3127, P.O. Box 3660, Riyadh 11481, Kingdom of Saudi Arabia, tel: +966 (11) 4295269 (office),

fax: +966 (11) 4299999, ext. 95276, e-mail: drahmedal@gmail.com; ahmedal@ksau-hs.edu.sa 
1732, 1743, and 1753 and were first illustrated by J.D. Santorini in 1775 [18].

The rugae palatinae are stratified squamous, mainly parakeratinised, epithelium on a connective tissue base that is deeply imbedded between the submucosa fatty tissue and the stratum reticulum of the palatum [24, 31]. The structure of the connective tissue of the rugae palatinae is different from the adjacent palatine tissues as its core has delicate reticulin fibres and different numbers and sizes of fibroblasts [28]. Rugae palatinae develop as localised regions of epithelial proliferation and thickening even prior to the elevation of the palatal shelves [20]. The fibroblasts and collagen fibres then accumulate in the connective tissue below these thickened epithelia. The orientation is controlled anteroposteriorly by the core fibres within the curve and in concentric curves across the base of each ruga [14]. The shape is maintained throughout life mainly due to the presence of these fibres and the existence of glycosaminoglycans, which cause the tissue to swell due to their hydrophilic nature [32]. The development of rugae consists of several phases. In the initial stage, at the time of their elevation, they have a relatively prominent appearance and occupy most of the length of the palatal shelves [12]. In a later stage, i.e., $55 \mathrm{~mm}$, they are presented as ridges where the anterior ones begin at the median raphe while the others are situated laterally [18]. The rugae patterns then become less regular towards the end of intra-uterine life with the disappearance of the posterior rugae accompanied by compression and the enhancement of prominence of the anterior rugae [18]. At birth, the form and orientation pattern of the rugae palatinae that are typical for the individual appear [23]. Nevertheless, their length continues to change during palatine growth, but their position remains the same throughout life [32]. Hence, it has been determined that they develop their typical orientation pattern at birth, acquire their final shape in adolescence and then remain stable throughout the individual's life [18].

Rugae palatinae have been found to differ among different populations and ethnic groups [14]. These differences give rise to the question, how much is due to genetic factors and how much is attributable to various environmental factors. Studies among twins have indicated that there is genetic basis expressed as similarity, though not identical, in rugae patterns [18]. Moreover, the rugae's numbers and arrangements are found to be species specific among mammals [22]. Therefore, with respect to palata rugae, it has been postulated that the orientation of the collagen fibres during embryogenesis and postnatal growth as well as the patterning of rugae in different populations are strictly controlled by genetics $[17,20]$. However, various environmental factors such as extreme finger sucking during infancy and persistent pressure following orthodontic treatment were also found to affect rugae [14]. The results regarding the use of rugae to predict sex have been inconsistent not only between populations but even among the same populations. For example, in a study conducted among two Indian populations, different rugae patterns did not show any sexual dimorphism [20], while a study conducted using the western Indian population as its sample revealed significant differences between the sexes based on types and numbers of rugae [10]. Moreover, while the Greeks exhibit an absence of sexual differences with respect to main ridge numbers, the Swazi population is found to express differences [12]. Thus, it is concluded that differences exist in the rugae patterns and in the expression of sexual dimorphism among different populations.

Sudan is a country with extensive indigenous diversity. The Sudanese Nubians have been the focus of anthropologic and archeologic studies due to their location in the Nile valley and because they are one of the oldest civilizations in Africa. However, there are few studies about the current Nubian population, which is an important ethnic group in contemporary Sudan. Moreover, there are no published reports about the rugae of the Sudanese population as most studies on rugae involve a limited range of global populations, e.g., Indians, South Africans, Egyptians, Saudis, Japanese, and Australians. Hence, the present study is conducted to determine the prevalence of the different biometric characteristics of the rugae palatinae in the Sudanese Nubians, to explore the existence of asymmetry among the rugae palatinae, and to determine the effectiveness of rugae palatinae in correctly identifying sex using logistic regression. This study was undertaken under the presumption it will provide valuable data for any further comparative study involving other Sudanese, Sub-Saharan Africans, or Northern Africans, such as Egyptian Nubians, and seeking to assess differences and to identify the impacts of various environmental factors. Accordingly, the findings of this study may be of immense help in establishing identity. Furthermore, to the best of the authors' knowledge, this study is the first to use logistic regression to assess the possibility of sex assignment using both continuous (dimension and orientation) and discrete (shapes) variables. 


\section{MATERIALS AND METHODS}

The study sample was comprised of 100 dental casts ( 50 from males and 50 from females). The age group of the studied sample ranged from 18 to 23 years, and all subjects were students from various universities located in Khartoum. Additionally, all subjects were Sudanese Nubians dating back at least three generations on both the mother's side and the father's side. They participants were administered a questionnaire to ascertain age, sex, ethnicity (up to three generations) and health issues. Any subject with a congenital anomaly/malformation, a chronic illness that might affect growth, a pathology/trauma to the palatum, severe malocclusion, orthognathic/orthodontic treatment, palatal surgery, active lesions, bony or soft tissue protuberances, or an allergy to the impression materials were excluded. This study was approved by the ethical committee of the Faculty of Medicine, University of Khartoum.

Informed consent was obtained from all subjects following an explanation regarding the nature of the study and the procedures that would be followed. To record the rugae, alginate impressions of the maxillary arch were obtained using chromatic alginate impression material (Alginmax) prepared as instructed by the manufacturer. The dental casts were made using type IV dental stone (Zermack) to obtain greater strength and accuracy. A plaster base was positioned for each cast to preserve the cast model. The rugae outlines were delineated under spotlight using a magnifying glass and a sharp $0.1 \mathrm{HB}$ graphite pencil. All rugae patterns were then assessed and measurements were conducted using a digital sliding calliper and a protractor (for angles).

The rugae classification was recorded based on the classification systems described by Lysell [18] and Thomas and Kotze's [29, 30]. The palatine rugae lengths were measured on each side to an accuracy of $0.01 \mathrm{~mm}$ using a digital sliding calliper. Based on their length, the rugae were divided into three categories: primary rugae $(>5 \mathrm{~mm})$, secondary rugae ( 3 to $5 \mathrm{~mm}$ ), and fragmentary rugae ( 2 to $3 \mathrm{~mm}$ ). Rugae less than $2 \mathrm{~mm}$ were disregarded. The shape of the rugae was classified into 5 major types: straight, curved, circular, wavy, and angular, and the united rugae were categorised as unified, branching, and cross-linked. The unification was further subdivided into diverging and converging types. The branching type had a branch that extended $1 \mathrm{~mm}$ or more from its origin in a lateral direction. The cross-linked type denotes a situation when 2 rugae join at a right angle. Any rugae shape that did not fit into this classification was regarded as non-specific. The direction of each primary ruga was classified according to the angle between the line joining its point of origin and point of termination with a line perpendicular to the median raphae. Forward-directed rugae were regarded as positive angles, backward-directed rugae were regarded as negative angles, and perpendicular rugae were regarded as zero angles. The direction of the branched rugae was determined at their termination in the midpoint between the ends of the two branches.

\section{Statistical analysis}

Prior to the recording of the findings, rugae observations were repeated twice for 20 casts to assess intra-observer error in interpretations. The discrepancies were minimal $(p>0.05)$. Thus, the total numbers of various rugae were counted based on classification and percentages were recorded. To explore sexual differences, the association between lengths and orientation and sex was assessed using the Mann-Whitney test as it does not require the assumption of normal distribution and has the statistical strength of the unpaired t-test. The association between rugae shape and sex was tested using $\chi^{2}$ analysis and, bilateral differences were assessed using Wilcoxon signed ranks tests. Using the Statistical Package for the Social Sciences (SPSS), version 14-computer software (SPSS, Inc., Chicago, IL, USA), a logistic regression analysis was used to assess the possibility of sex prediction using the discrete variable (shape) and the continuous variables (dimensions and orientation) [15]. In a logistic regression analysis, coefficients and constants are derived and logit is then calculated by multiplying the variables with their respective coefficients, which are then added to the constant. The resultant logit is used to calculate the predictive probability where an individual with a predictive probability $<0.5$ is classified as female and a predictive probability $>0.5$ is classified as male.

\section{RESULTS}

The numbers and percentages of different rugae lengths and directions in both sexes are presented in Table 1. Males have a larger number of rugae compared to females. While the mean number of primary rugae exceeds that of females, the females have more secondary rugae than males. The fragmented rugae are almost equal between the sexes. Nonetheless, primary, which are sexually dimorphic, are dominant in both sexes compared to secondary and fragmented rugae. The most common direction of the rugae is the forward-directed, which is followed by the backward-directed and then by the perpendicular-directed in both sexes. Males tend to have more forward-directed rugae and fewer perpendicular-directed rugae compared with females, but there exist no statistically significant differences between the sexes. 
Table 1. Descriptive statistics for palatal rugae lengths and directions in both sexes

\begin{tabular}{|c|c|c|c|c|c|c|c|c|c|c|}
\hline \multirow[t]{2}{*}{ Variable } & \multicolumn{4}{|c|}{ Males } & \multicolumn{4}{|c|}{ Females } & \multicolumn{2}{|c|}{ Mann-Whitney test } \\
\hline & Number & $\%$ & Mean & SD & Number & $\%$ & Mean & SD & $\mathbf{U}$ & $\mathbf{P}$ \\
\hline \multicolumn{11}{|l|}{ Length } \\
\hline Primary & 488 & 73.94 & 9.76 & 1.92 & 453 & 71.45 & 9.06 & 1.57 & 952.500 & $0.037^{*}$ \\
\hline Secondary & 130 & 19.70 & 2.60 & 2.00 & 138 & 21.77 & 2.76 & 1.44 & 1124.500 & 0.377 \\
\hline Fragmented & 42 & 6.36 & 0.84 & 0.98 & 43 & 6.78 & 0.86 & 0.93 & 1215.000 & 0.796 \\
\hline \multicolumn{11}{|l|}{ Direction } \\
\hline Forward & 373 & 56.52 & 7.46 & 3.12 & 339 & 53.47 & 6.78 & 2.19 & 1103.500 & 0.307 \\
\hline Backward & 243 & 36.82 & 4.86 & 2.50 & 242 & 38.17 & 4.84 & 2.35 & 1224.500 & 0.859 \\
\hline Perpendicular & 44 & 6.67 & 0.88 & 0.85 & 53 & 8.36 & 1.06 & 0.10 & 1141.000 & 0.426 \\
\hline
\end{tabular}

${ }^{*}$ Significant at level $\mathrm{p}<0.05 ; \mathrm{SD}$ - standard deviation

Table 2. Descriptive statistics for palatal rugae shapes in both sexes

\begin{tabular}{|c|c|c|c|c|c|c|c|c|c|c|c|}
\hline \multirow[t]{2}{*}{ Shape } & \multicolumn{4}{|c|}{ Males } & \multicolumn{4}{|c|}{ Females } & \multicolumn{3}{|c|}{ Chi-square test } \\
\hline & Number & $\%$ & Mean & SD & Number & $\%$ & Mean & SD & $\chi^{2}$ value & $\begin{array}{c}\text { Degree } \\
\text { of freedom }\end{array}$ & $\mathbf{P}$ \\
\hline Straight & 159 & 24.09 & 3.18 & 2.48 & 158 & 24.92 & 3.16 & 1.61 & 10.106 & 9 & 0.342 \\
\hline Curve & 183 & 27.73 & 3.66 & 1.65 & 172 & 27.13 & 3.44 & 1.92 & 6.710 & 9 & 0.667 \\
\hline Wavy & 194 & 29.39 & 3.88 & 1.80 & 176 & 27.76 & 3.52 & 1.40 & 11.194 & 8 & 0.191 \\
\hline Annular & 18 & 2.73 & 0.36 & 0.56 & 20 & 3.15 & 0.40 & 0.57 & 0.194 & 2 & 0.908 \\
\hline Branch & 43 & 6.52 & 0.86 & 0.93 & 37 & 5.84 & 0.74 & 0.80 & 1.150 & 4 & 0.886 \\
\hline Crosslink & 11 & 1.67 & 0.22 & 0.46 & 13 & 2.05 & 0.26 & 0.73 & 0.346 & 2 & 0.841 \\
\hline Angular & 14 & 2.12 & 0.28 & 0.50 & 20 & 3.15 & 0.40 & 0.70 & 2.099 & 3 & 0.552 \\
\hline Non specific & 16 & 2.42 & 0.32 & 0.68 & 6 & 0.95 & 0.12 & 0.33 & 6.590 & 2 & $0.037^{*}$ \\
\hline Converging & 9 & 1.36 & 0.18 & 0.39 & 18 & 2.84 & 0.36 & 0.48 & 4.110 & 1 & $0.043^{*}$ \\
\hline Diverging & 13 & 1.97 & 0.26 & 0.49 & 14 & 2.21 & 0.28 & 0.50 & 0.057 & 2 & 0.972 \\
\hline
\end{tabular}

${ }^{*}$ Significant at level $p<0.05 ; S D-$ standard deviation

The different shapes of rugae are observed and found to present varying degrees of dominance, as presented in Table 2. The wavy, curved and straight rugae are the most prevalent forms in both sexes, while the converging and non-specific shapes are the only sexually dimorphic shapes. The least common form in males is the converging unification, while females exhibit fewer non-specific shapes than males and both shapes are sexually dimorphic.

The bilateral differences in rugae lengths, directions, and shapes in both sexes were assessed, and the results are presented in Tables 3 and 4 . The results show that there is significant asymmetry in the primary rugae of females, while the primary and fragmented rugae tend to be located on the left side in both sexes. Both males and females show significant asymmetry in forward-directed and backward-directed rugae, while perpendicular-directed rugae are not significant. The only significant diffe- rences between sides with respect to shapes is found in the divergent shape in males $(p<0.035)$ and the wavy shape in females $(p<0.041)$.

The possibility of assigning sexes using a logistic regression analysis is assessed using the combination of all continuous variables (lengths and directions) and discrete variables. None of the continuous variables demonstrate any significant differences (Table 5). The prediction for males is better than that for females (64\% compared to $56 \%$ ), as shown in Table 6 . The shapes are logistically regressed to assess their effectiveness as sex predictors and the results are presented in Table 7. As evidenced from the data in Table 7, only converging unification expresses minimal sexual dimorphism $(p<0.046)$. Sex prediction increased slightly, to $65 \%$, with male prediction remains slightly better than female prediction $(68 \%$ and $62 \%$, respectively) (Table 8 ). 
Table 3. Bilateral differences (left-right) in rugae lengths and directions in both sexes

\begin{tabular}{|c|c|c|c|c|c|c|}
\hline \multirow[t]{2}{*}{ Variable } & \multicolumn{3}{|c|}{ Males } & \multicolumn{3}{|c|}{ Females } \\
\hline & $\begin{array}{c}\text { Right } \\
\text { Mean } \pm \text { SD }\end{array}$ & $\begin{array}{c}\text { Left } \\
\text { Mean } \pm \text { SD }\end{array}$ & $\mathbf{P}^{*}$ & $\begin{array}{c}\text { Right } \\
\text { Mean } \pm \text { SD }\end{array}$ & $\begin{array}{c}\text { Left } \\
\text { Mean } \pm \text { SD }\end{array}$ & $\mathbf{P}^{*}$ \\
\hline \multicolumn{7}{|l|}{ Lengths } \\
\hline Primary & $4.86 \pm 1.40$ & $4.90 \pm 1.30$ & 0.745 & $4.36 \pm 1.00$ & $4.70 \pm 0.91$ & 0.041 \\
\hline Secondary & $1.40 \pm 1.26$ & $1.20 \pm 1.36$ & 0.457 & $1.38 \pm 1.12$ & $1.38 \pm 1.03$ & 0.873 \\
\hline Fragmented & $0.40 \pm 0.64$ & $0.44 \pm 0.61$ & 0.729 & $0.40 \pm 0.67$ & $0.46 \pm 0.58$ & 0.627 \\
\hline \multicolumn{7}{|l|}{ Direction } \\
\hline Forward & $2.80 \pm 1.74$ & $4.66 \pm 2.23$ & 0.000 & $2.46 \pm 1.42$ & $4.32 \pm 1.66$ & 0.000 \\
\hline Backward & $3.36 \pm 1.84$ & $1.50 \pm 1.36$ & 0.000 & $3.22 \pm 1.52$ & $1.62 \pm 1.44$ & 0.000 \\
\hline Perpendicular & $0.50 \pm 0.74$ & $0.38 \pm 0.64$ & 0.461 & $0.46 \pm 0.65$ & $0.60 \pm 0.76$ & 0.346 \\
\hline
\end{tabular}

*Wilcoxon signed ranks test; SD — standard deviation

Table 4. Bilateral differences (left-right) in rugae shapes in both sexes

\begin{tabular}{|c|c|c|c|c|c|c|}
\hline \multirow[t]{2}{*}{ Variable } & \multicolumn{3}{|c|}{ Males } & \multicolumn{3}{|c|}{ Females } \\
\hline & $\begin{array}{c}\text { Right } \\
\text { Mean } \pm \text { SD }\end{array}$ & $\begin{array}{c}\text { Left } \\
\text { Mean } \pm \text { SD }\end{array}$ & $\mathbf{P}^{*}$ & $\begin{array}{c}\text { Right } \\
\text { Mean } \pm \text { SD }\end{array}$ & $\begin{array}{c}\text { Left } \\
\text { Mean } \pm \text { SD }\end{array}$ & $\mathrm{P}^{*}$ \\
\hline Straight & $1.62 \pm 1.48$ & $1.56 \pm 1.57$ & 0.735 & $1.50 \pm 1.28$ & $1.66 \pm 1.04$ & 0.549 \\
\hline Curve & $1.94 \pm 1.25$ & $1.72 \pm 1.36$ & 0.388 & $1.74 \pm 1.18$ & $1.70 \pm 1.25$ & 0.687 \\
\hline Wavy & $1.84 \pm 1.13$ & $2.04 \pm 1.23$ & 0.385 & $1.54 \pm 1.03$ & $1.98 \pm 1.00$ & 0.041 \\
\hline Annular & $0.18 \pm 0.44$ & $0.18 \pm 3.90$ & 1.000 & $0.18 \pm 3.90$ & $0.22 \pm 1.42$ & 0.617 \\
\hline Branch & $0.36 \pm 0.56$ & $0.50 \pm 0.71$ & 0.273 & $0.46 \pm 0.71$ & $0.28 \pm 0.50$ & 0.155 \\
\hline Crosslink & $0.10 \pm 0.30$ & $0.12 \pm 0.33$ & 0.739 & $0.14 \pm 0.41$ & $0.12 \pm 0.33$ & 0.782 \\
\hline Angular & $0.14 \pm 0.35$ & $0.14 \pm 0.35$ & 1.000 & $0.18 \pm 0.44$ & $0.22 \pm 0.51$ & 0.635 \\
\hline Non-specific & $0.18 \pm 0.39$ & $0.14 \pm 0.35$ & 0.317 & $0.06 \pm 0.24$ & $0.06 \pm 0.24$ & 1.000 \\
\hline Converging & $0.10 \pm 0.30$ & $0.08 \pm 0.27$ & 0.739 & $0.16 \pm 0.37$ & $0.20 \pm 0.40$ & 0.637 \\
\hline Diverging & $0.20 \pm 0.40$ & $0.06 \pm 0.24$ & 0.035 & $0.18 \pm 3.90$ & $0.10 \pm 0.30$ & 0.248 \\
\hline
\end{tabular}

*Wilcoxon signed ranks test; SD — standard deviation

Table 5. Regression co-efficient (b) of all lengths and directions

\begin{tabular}{lccc}
\hline SI. No. & Variables & b & P \\
\hline 1 & Primary & -0.037 & 0.888 \\
2 & Secondary & -0.282 & 0.267 \\
3 & Fragmented & -0.269 & 0.430 \\
4 & Forward & 0.285 & 0.251 \\
5 & Backward & 0.243 & 0.291 \\
& Constant (a) & -1.877 & 0.145 \\
\hline
\end{tabular}

\section{DISCUSSION}

Rugae palatinae are important in anatomy and dentistry, and it has been suggested that they serve as an alternative method of identification in the absence of other definitive techniques, e.g., DNA analysis, finger-printing, and dental recording [5].

The findings of this study indicate that primary rugae are the most dominant, followed by the secondary rugae. Furthermore, males have more total rugae than females, and there is a significant difference between the sexes in the number of primary rugae. Proportionally, males have a higher percentage of primary rugae than their female counterparts, while the reverse is true with respect to secondary rugae. The means of primary rugae are 9.76 for males and 9.06 for females. These results are close to the findings regarding Indian males and females (9.7 and 9.2) [13], but higher than those for Jordanians [19], Rwandans [11], Saudis [9], Japanese [8], Caucasian Australians [14], 
Table 6. Predictive value using lengths and directions

\begin{tabular}{lccccc}
\hline SI. No. & Actual group & \multicolumn{2}{c}{ Predicted value } & Total No. & Correct \\
\cline { 3 - 4 } & & Female $(\mathbf{n}=\mathbf{5 0})$ & Male $(\mathbf{n}=\mathbf{5 0})$ & & $64 \%$ \\
\hline 1 & Female & 32 & 18 & 50 & $56 \%$ \\
2 & Male & 22 & 28 & 50 & $60 \%$ \\
\hline
\end{tabular}

Table 7. Regression co-efficient (b) of all shapes

\begin{tabular}{lccc}
\hline SI. No. & Variables & (b) & P \\
\hline 1 & Straight & 0.006 & 0.953 \\
2 & Curve & 0.075 & 0.573 \\
3 & Wavy & 0.167 & 0.304 \\
4 & Annular & -0.128 & 0.755 \\
5 & Branch & 0.189 & 0.517 \\
6 & Crosslink & -0.097 & 0.835 \\
7 & Angular & -0.347 & 0.370 \\
8 & Converging & -1.021 & 0.046 \\
9 & Diverging & 0.133 & 0.778 \\
& Constant (a) & -0.632 & 0.642 \\
\hline
\end{tabular}

and Nepalese [13]. Nevertheless, they are still lower than those reported for the Australian Aboriginals [14]. This variation in the number of rugae among populations may be due to the genetic factors affecting the breadth of the palatum and, to lesser degree to, environmental factors $[14,26]$. The determination that males have more primary rugae than females can be attributed to the extra period of growth among males yielding broader palati [7]. The only significant difference between the sexes is found in the number of primary rugae. This finding differs from the findings reported for Jordanians [19] and Australian Aboriginals [14], but is consistent with the results for western Indians [10]. Furthermore, the most prevalent rugae direction in Sudanese Nubians is forward, which is followed by backward and then perpendicular for both sexes. Forward-directed rugae represent the majority in both sexes with the percentage in males (56.52\%) exceeding that of females $(53.47 \%)$, while the percentages of backward-directed and perpendicular-directed rugae are slightly higher for females. This finding is in accordance with the findings for Jordanians [19], but it contradicts the results related to Rwandans [11] where females have more forward-directed rugae. While the results of this study show that there are no significant sexual differences in the orientation of rugae, a previous study of Jordanians found sexual dimorphism in forward-directed rugae [19].

The present study finds that all shapes used in this study are present in Sudanese Nubians, though there is existing variability with respect to dominance. This is in contrast to the findings regarding Saudi children as they lack the crosslink shape. The most prevalent are the wavy, curved and straight rugae, in descending order, as they account for $81.21 \%$ of the rugae in males and $79.81 \%$ in females, while converging unification is the least prevalent in males and crosslink is the least prevalent in females. Furthermore, the degree of complexity is more evident in males as 16 rugae could not be assigned to one of the used forms, a phenomenon that occurred with only 6 rugae in females. This finding indicates the need for describing/identifying other shapes and conducting a larger scale study. The findings of this study confirm previous findings with respect to Egyptian and Saudi children [1], Saudi adults [9], and Indians [13, 21, 26] where wavy and

Table 8. Predictive value using all shapes

\begin{tabular}{lccccc}
\hline SI. No. & Actual group & \multicolumn{2}{c}{ Predicted value } & Total No. & Correct \\
\cline { 3 - 4 } & & Female $(\mathbf{n}=\mathbf{5 0})$ & Male $(\mathbf{n}=\mathbf{5 0})$ & & $62 \%$ \\
\hline 1 & Female & 31 & 19 & 50 & $68 \%$ \\
2 & Male & 16 & 34 & 50 & $65 \%$ \\
\hline
\end{tabular}


curved rugae are the most common shapes followed by straight rugae. The results are also consistent with those reported for Australian Aborigines and Caucasian populations as they, too, are found to have a higher prevalence of wavy- and curved-shaped rugae [14]. Departing from this trend, however, wavy-shaped rugae are the most prevalent followed by diverging rugae in Jordanians [19], and wavy followed by straight and curved are the most prevalent among Rwandans [11] and Nepalese [13]. Despite the fact that the wavy shape seems to be the most dominant pattern in most studied populations, the variability in percentages and the order of other shapes present indicates the need for population-specific studies. Regardless, the results of this study indicate that there is no sexual dimorphism in most rugae shapes $(p>0.05)$ apart from converging unification and non-specific shapes. This finding is consistent with those related to Saudi adults. That is, converging shapes are found to be sexually dimorphic and higher in females [9]. However, shapes did not show any sexual dimorphism among Jordanians [19], Rwandans [11], Egyptians or Saudi children [1]. This may be attributable to the classification used regarding the shapes used in the study, as it indicates that non-specific rugae are sexually dimorphic while converging unified rugae differ significantly between males and females in Sudanese Nubians and Saudi adults [9]. It is also possible, however, that the degrees of sexual differences in rugae patterns are ethnic specific or that the results are due to individual differences.

The present study finds that the left side, for both sexes, tends to have more primary rugae. This observation is the same as the findings reported for Australian Aborigines [14], Swedes [12], Rwandans [11], Mysoreans [26], and Japanese [8]. On the other hand, the right side is found to have more secondary rugae than the left side in males, while females tend to have approximately the same amount on both sides. This finding supports the assumption that there is an indirect relation between primary and secondary rugae [7]. The findings of this study further indicate that there are no significant differences between the right and left sides when comparing the means of the rugae palatinae dimensions, except for primary rugae $(p<0.041)$ in females. Most of the shapes show no significant bilateral differences, except for the diverging shape in males $(p<0.035)$ and the wavy shape in females $(p<0.041)$, and the orientations express the maximum degree of asymmetry in forward and backward directions for both sexes $(p<0.001)$. Forward-directed rugae are more common on the left side in both sexes, whereas backward-directed rugae are more common on the right side in both sexes. This asymmetry in directions contradicts the assumption that the development of rugae is a coordinated process that occurs throughout the palatum, thus indicating that differential growth rates exist between sides in both males and females.

Owing to the apparent lack of systematic trends in sex between populations, some authors conclude that rugae do not possess discriminatory ability. To ascertain this, we subjected the rugae dimensions, directions, and shapes to logistic regression analysis, which was used in preference to the commonly used discriminant function analysis as the former can be used for continuous and discrete variables without the assumptions of normal distribution, linearity, or equity of variance within each group [15]. Furthermore, some authors suggest that using of non-parametric discriminant procedure is more appropriate $[15,20]$. In our study, the accuracy with which the logistic regression equation can identify sex using the dimensions and directions is $56 \%$ and $64 \%$, respectively, for males and females. In contrast, the shapes yield better prediction results for both sexes (62\% for females and $68 \%$ for males). Using logistic regression analysis, previous study among Indians have determined sex at a $99.2 \%$ success rate using shapes in 120 subjects [25]. Additionally, discriminant function analyses obtained a $73.08 \%$ success rate using shapes and dimensions in 100 coastal Andhra Indians, and a $78 \%$ success rate using only dimensions [6]. Another study conducted among two groups of Indians shows no sexual dimorphism [20]. The findings of this present study reveal a low success rate among Sudanese Nubians compared to the two aforementioned studies, which may indicate ethnic variability in the expression of sexual dimorphism using rugae palatinae as the majority of the previous studies among Indians indicated a lack of sexual dimorphism in rugae palatinae, e.g., Puducherry [16]. Moreover, the high success rate in these two studies may be due to the existence of individually related differences. Previous studies of the Sudanese reveal moderate to high degrees of sexual dimorphism when using limbs $[2,3]$ and crania [4]. The results of this study, including the dimensions, shapes and directions of rugae palatinae, lack this discriminatory ability, thus indicating that the rugae palatinae is not an effective tool for the assigning of sex and signifying the need to assess the discriminative capability of other aspects of Sudanese Nubians. As this study establishes baseline data for any large scale study of the Sudanese and the Southern Sudanese, it can be used for comparative purposes among neighbouring populations as well as on a global scale. 


\section{CONCLUSIONS}

The dimensions, shapes, and orientations of the rugae palatinae were analysed in Sudanese Nubians. While the rugae of Sudanese Nubians exhibited all shapes included in the study, the most dominant was the primary rugae and the most prevalent shapes were the wavy, curved, and straight forms in both sexes. The predominant direction was forward-directed rugae. Furthermore, while there was no sexual dimorphism in rugae orientation, primary and converging rugae demonstrated significant differences between the sexes. The dimensions and shapes expressed the least asymmetry between sides when compared with orientation as forward and backward directions were found to be asymmetrical. The primary rugae and wavy shapes in females and the diverging shapes in males exhibited asymmetry. It is concluded that based on the findings of this study, the use of rugae palatinae to assign sex among Sudanese Nubians is not recommended. The possible differences found in the rugae palatinae with respect to the dimensions, shapes, and directions in different regions and among different ethnicities within the same population necessitate further studies involving larger samples. Moreover, further shapes require classification.

\section{REFERENCES}

1. Abdellatif AM, Awad SM, Hammad SM (2011) Comparative study of platal rugae shapes in two samples of Egyptians and Saudi children. Pediatric Dental J, 21: 123-128.

2. Ahmed AA (2013) Estimation of sex from the lower limb measurements of Sudanese adults. Forensic Sci Int, 229: 169e161-167.

3. Ahmed AA (2013) Estimation of sex from the upper limb measurements of Sudanese adults. J Forensic Leg Med, 20: 1041-1047.

4. Ahmed AA, Mohammed AA, Hassan MA (2011) Sex determination from cranial measurements in recent northern Sudanese. Khartoum Med J, 4: 539-547.

5. Barbieri AA, Scoralick RA, Naressi SC, Moraes ME, Daruge E Jr., Daruge $E$ (2013) The evidence of the rugoscopy effectiveness as a human identification method in patients submitted to rapid palatal expansion. J Forensic Sci, 58 (suppl. 1): S235-S238.

6. Bharath ST, Kumar GR, Dhanapal R, Saraswathi T (2011) Sex determination by discriminant function analysis of palatal rugae from a population of coastal Andhra. J Forensic Dent Sci, 3: 58-62.

7. Dawasaz A, Dinkar AD (2013) Rugoscopy: predominant pattern, uniqueness, and stability assessment in the Indian goan population. J Forensic Sci, 58: 1621-1627.

8. Dohke M, Osato S (1994) Morphological study of the palatal rugae in Japanese 1. Bilateral differences in the regressive evaluation of the palatal rugae. Japanese J Oral Biol, 36: 126-140.

9. Fahmi FM, Al-Shamrani SM, Talic YF (2001) Rugae pattern in a Saudi population sample of males and females. Saudi Dental J, 13: 92-95.

10. Gondivkar SM, Patel S, Gadbail AR, Gaikwad RN, Chole R, Parikh RV (2011) Morphological study of the palatal rugae in western Indian population. J Forensic Leg Med, 18: 310-312.
11. Goyal S, Goyal S (2013) Study of platal rugae pattern of Rwandan patients attending the dental department at King Faisal hospital, Kigali, Rwanda: a preliminary study. RMJ, 70: 19-25.

12. Hauser G, Daponte A, Roberts MJ (1989) Palatal rugae. J Anat, 165: 237-249.

13. Kallianpur S, Desai A, Kasetty S, Sudheendra U, Joshi P (2011) An anthropometric analysis of facial height, arch length, and palatal rugae in the Indian and Nepalese population. J Forensic Dent Sci, 3: 33-37.

14. Kapali S, Townsend G, Richards L, Parish T (1997) Palatal rugae patterns in Australian aborigines and Caucasians. Aust Dent J, 42: 129-133.

15. Kotrashetti VS, Hollikatti K, Mallapur MD, Hallikeremath SR, Kale $A D$ (2011) Determination of palatal rugae patterns among two ethnic populations of India by logistic regression analysis. J Forensic Leg Med, 18: 360-365.

16. Kumar S, Vezhavendhan N, Shanthi V, Balaji N, Sumathi MK, Vendhan P (2012) Palatal rugoscopy among Puducherry population. J Contemp Dent Pract, 13: 401-404.

17. Luke DA (1988) Development and growth of palatal rugae in the mouse. Acta Anat (Basel), 133: 41-44.

18. Lysell $L$ (1955) Plicae palatinae transversae and papilla incisiva in man: a morphologic and genetic study. Acta Odontologica Scand, 13: 1-137.

19. Mustafa AG, Allouh M, Tarawneh I, Alrbata R (2013) Morphometric analysis of palatal rugae among Jordanians: further evidence of worldwide palatal rugae individuality. Austr J Forensic Scien, 46: 53-63.

20. Nayak P, Acharya AB, Padmini AT, Kaveri H (2007) Differences in the palatal rugae shape in two populations of India. Arch Oral Biol, 52: 977-982.

21. Paliwal A, Wanjari S, Parwani R (2010) Palatal rugoscopy: Establishing identity. J Forensic Dent Sci, 2: 27-31.

22. Pantalacci S, Prochazka J, Martin A, Rothova M, LambertA, Bernard L, Charles C, Viriot L, Peterkova R, Laudet V (2008) Patterning of palatal rugae through sequential addition reveals an anterior/ posterior boundary in palatal development. BMC Dev Biol, 8: 116.

23. Patil MS, Patil SB, Acharya AB (2008) Palatine rugae and their significance in clinical dentistry: a review of the literature. J Am Dent Assoc, 139: 1471-1478.

24. Peavy DC, Jr., Kendrick GS (1967) The effects of tooth movement on the palatine rugae. J Prosthet Dent, 18: 536-542.

25. Saraf A, Bedia S, Indurkar A, Degwekar S, Bhowate R (2011) Rugae patterns as an adjunct to sex differentiation in forensic identification. J Forensic Odontostomatol, 29: 14-19.

26. Shetty SK, Kalia S, Patil K, Mahima VG (2005) Palatal rugae pattern in Mysorean and Tibetan populations. Indian J Dent Res, 16: 51-55.

27. Simmons JD, Moore RN, Erickson LC (1987) A longitudinal study of anteroposterior growth changes in the palatine rugae. J Dent Res, 66: 1512-1515.

28. Thomas CJ (1984) The prenatal developmental microscopic anatomy of the palatal rugae. J Dent Assoc S Afr, 39: 527-533.

29. Thomas CJ, Kotze TJ (1983) The palatal rugae pattern in Southern African human populations. Part I. A description of the populations and a method for its investigation. J Dent Assoc S Afr, 38: 547-553.

30. Thomas CJ, Kotze TJ (1983) The palatal rugae pattern: a new classification J Dent Assoc S Afr, 38: 153-157.

31. Thomas CJ, Rossouw RJ (1991) The early development of palatal rugae in the rat. Aust Dent J, 36: 342-348.

32. Thomas C, Van Wyk CW (1987) Elastic fibre and hyaluronic acid in the core of human palatal rugae. J Biol Buccale, 15: 171-174. 